\title{
Marcadores de selección en cerdos Pampa Rocha: comparación con razas autóctonas de España y Portugal
}

\author{
Carmen Burgos $\mathrm{S}^{1} \otimes(\mathbb{0})$ Ph.D; Silvia Llambí $\mathrm{D}^{2} \otimes \mathbb{0}$ Ph.D; Jorge Hidalgo $\mathrm{G}^{1} \otimes(\mathbb{0}$ M.Sc; \\ María Montenegro S ${ }^{凶}$ M.Sc; Victoria Arruga L ${ }^{3} \bowtie(0)$ Ph.D; Pascual López-Buesa ${ }^{*} \bowtie \mathbb{C}$ Ph.D.
}

\begin{abstract}
${ }^{1}$ Universidad de Zaragoza. Facultad de Veterinaria, Departamento de Producción Animal y Ciencia de los Alimentos. Miguel Servet 177, C.P. 50013, Zaragoza, España.

2Universidad de la República, Facultad de Veterinaria, Departamento de Genética y Mejora Animal. Lasplaces 1550. C.P. 11600, Montevideo, Uruguay.

3Universidad de Zaragoza. Facultad de Veterinaria, Laboratorio de Citogenética y Genética Molecular. Miguel Servet 177, C.P. 50013, Zaragoza, España.

*Corresponding author: plopezbu@unizar.es
\end{abstract}

Received: October 2018; Accepted: March 2019; Published: April 2019.

\section{RESUMEN}

Objetivo. El análisis de marcadores de selección permite obtener datos de la vida evolutiva de una raza o línea y permite también evaluar la conveniencia o no de su uso en programas de mejora genética. Hemos evaluado SNPs en cuatro genes (IGF2, MC4R, PRKAG3 y PEPCK-C), que tienen importantes efectos fenotípicos, en cerdos de la raza Pampa Rocha, una raza criolla, y hemos comparado sus frecuencias alélicas con cerdos de diversas razas autóctonas y líneas de España y Portugal no sometidas a selección así como con jabalíes y cerdos de la raza Piétrain. Materiales y métodos. Los SNPs fueron analizados mediante diversa técnicas de RT-PCR. Resultados. Los resultados de los análisis muestran una similitud de frecuencias alélicas entre los cerdos de la raza Pampa Rocha y los cerdos autóctonos de la península ibérica sobre todo en el gen IGF2 y, en menor medida en el gen PEPCK-C. Sin embargo difieren considerablemente en el caso del marcador MC4R y, también en menor medida, en PRKAG3. En el trabajo se discute el uso potencial de los resultados obtenidos para orientar la selección genética de cerdos de la raza Pampa Rocha. Conclusiones. Nuestros resultados demuestran la peculiaridad de la raza Pampa Rocha con respecto a los marcadores estudiados.

Palabras clave: IGF2, MC4R, PRKAG3, PEPCK, Sus scrofa (Fuente: CAB, BioThesaurus).

\begin{abstract}
Objective. The analysis of selection markers allows to obtain information about the evolutive story of a particular breed or line and allows also to evaluate the usefulness of those markers for breeding programs. We have analyzed SNPs in four genes of the creole pig breed Pampa Rocha and we have compared their allelic frequencies with the allelic frequencies of diverse autochthonous breeds of Spain and Portugal and also with Piétrain pigs and wild boars. Materials and methods. The SNPs were analyzed using diverse RT-PCR methods. Results. The results of the analysis show that Pampa Rocha pigs have similar allelic frequencies with the autochthonous breeds of Spain and Portugal especially in the case of IGF2 and also, but not so coincident, in the case of PEPCK-C. However, they differ considerably for MC4R, and also, but in a lower extent, for PRKAG3. We discuss in this work the usefulness of our results for breeding of Pampa Rocha pigs. Conclusions. Our results demonstrate the peculiarity of the Pampa Rocha breed regarding the markers studied.
\end{abstract}

Keywords: IGF2, MC4R, PRKAG3, PEPCK, Sus scrofa (Source: CAB, BioThesaurus).

Como citar (Vancouver)

Burgos SC, Llambí DS, Hidalgo GJ, Montenegro SM; Arruga LV, López-Buesa P. Marcadores de selección en cerdos Pampa Rocha: comparación con razas autóctonas de España y Portugal. Rev MVZ Cordoba. 2019; 24(2):7198-7202. DOI: https://doi.org/10.21897/rmvz.1642

(7) (2) (C) (los) autor (es), Revista MVZ Córdoba 2019. Este artículo se distribuye bajo los términos de la licencia internacional Creative Commons Attribution 4.0 (https://creativecommons.org/licenses/by-sa/4.0/), que permite el uso sin restricciones, la distribución y la reproducción en cualquier medio, siempre que se otorgue el crédito apropiado al autor o autores originales y la fuente. 


\section{INTRODUCCION}

La selección genética realizada en el cerdo durante las últimas décadas ha estado enfocada a obtener animales magros. Un ejemplo claro del éxito de este proceso es la reducción de los índices de conversión desde valores de 4.3, e incluso superiores, en los años sesenta a valores inferiores a 2.2 en la actualidad en las razas más eficientes como la Piétrain (1). En este proceso se ha utilizado el espesor de tocino dorsal (BT) como criterio de selección. La reducción en el BT ha sido espectacular. Los valores que presentan actualmente las razas más seleccionadas se encuentran en el límite inferior fisiológico. Esta reducción en BT ha venido acompañada de una disminución en el contenido de grasa intramuscular (IMF). Como consecuencia de este descenso en IMF se han visto afectados negativamente algunos caracteres de calidad de la carne como la jugosidad, el sabor o la capacidad de retención de agua $(2,3)$. Los cerdos actuales son máquinas biológicas muy eficientes en la conversión de alimento en carne pero producen carnes de baja calidad sensorial.

Para contrarrestar esta tendencia, en algunos países industrializados de Europa se ha utilizado otra estrategia diferente: el uso de razas autóctonas no sometidas a selección para obtener productos cárnicos de alta calidad dirigidos a los mercados de productos de alta gama (4). El paradigma del éxito de esta estrategia es la raza ibérica, cuyos productos curados alcanzan precios muy elevados (un solo jamón curado de las marcas más prestigiosas puede llegar a costar hasta u\$788\$).

Los caracteres complejos, como IMF, son resultado de los efectos producidos por multitud de genes menores. Sin embargo, también existen algunos genes cuyos efectos sobre este carácter son especialmente pronunciados. Estos genes, denominados genes mayores, pueden emplearse para potenciar los fenotipos deseados. Los genes mayores conocidos implicados en la determinación del contenido graso (tanto IMF como BT) son escasos. Algunos de los mejor conocidos son RYR1, el gen del halotano, IGF2, factor de crecimiento de insulina2 (5), MC4R, receptor de melanocortina 4 (6), o PRKAG3, una subunidad reguladora de la proteína quinasa activada por AMP (7).

Recientemente fue descubierto un SNP en la PEPCK-C; la fosfoenolpiruvato carboxiquinasa citosólica, con notables efectos sobre BT e IMF y que van, además, en sentido opuesto (1). Estos marcadores pueden resultar muy útiles para varios fines: por un lado para investigar la historia de la selección llevada a cabo en una raza o para asignar individuos a una determinada raza y, por otro lado, para introgresarlos (en el caso de que ya estén presentes) en razas no seleccionadas para mejorar, principalmente, los caracteres productivos.

El cerdo Pampa Rocha es un cerdo criollo de Uruguay en cuyos orígenes se encuentran los introducidos por los colonizadores españoles y portugueses. Estos cerdos ya habían sido cruzados con poblaciones asiáticas introducidas unos cuantos siglos atrás para mejorar principalmente caracteres maternales como la prolificidad y posteriormente, los cerdos Pampa Rocha se cruzaron con las razas Berkshire y Poland China (8).
Las hembras Pampa Rocha presentan caracteres maternales y de capacidad de pastoreo muy favorables. Su cruce con machos Duroc produce una descendencia con unos parámetros de crecimiento y ganancia de peso mejorados. Los machos Duroc también se cruzan con hembras ibéricas para producir una mejora de los caracteres productivos (9). Los productos curados obtenidos a partir de cerdos de este cruce se encuentran entre los mejores productos ibéricos, siempre y cuando la línea Duroc utilizada no sea magra.

El objetivo del presente trabajo fue investigar la presencia de alelos asociados a la selección en cerdos Pampa Rocha. Las mutaciones IGF2 (5), MC4R (6), PRKAG3 (7) y PEPCK (1) fueron analizadas por diversos métodos y también fueron estudiadas en jabalís, cerdos Piétrain y algunas razas o líneas de cerdos autóctonas de España y Portugal. Finalmente se evaluó la utilidad de dicho análisis para mejorar el valor comercial de los cerdos de la raza Pampa Rocha.

\section{MATERIALES Y METODOS}

Material animal. Las muestras de músculo congelado de jabalí nos las proporcionaron algunos amigos cazadores de nuestro Departamento. Las muestras de músculo congelado de los cerdos ibéricos analizados procedían de la cooperativa ganadera COVAP (Pozoblanco, España) y del CENSYRA (Badajoz, España). El Dr. Carballo García (Universidad de Vigo, España) nos suministró las muestras de músculo congelado de los cerdos Porco Celta, así como las de pelo de los Bízaro. Las muestras de pelo de los cerdos Pampa Rocha se obtuvieron de animales pertenecientes a la Unidad de Producción de Cerdos del Centro Regional Sur-Facultad de Agronomía (Universidad de la República) situada en Progreso, Departamento de Canelones, Uruguay. La empresa Agropecuaria OBANOS S.A. (Marcilla, España) nos proporcionó el semen de los cerdos Piétrain analizados.

Extracción de DNA. El DNA se extrajo de músculo, semen o de folículos pilosos utilizando el kit RealPure Genomic DNA Extraction de Durviz (Valencia, España) 0 el kit GenomicPrep ${ }^{T M}$ DNA isolation de Amersham Biosciences (Little Chalfont, Reino Unido), de acuerdo con las instrucciones del fabricante. La concentración de DNA en el extracto se determinó utilizando un espectrofotómetro NanoDrop 1000 (Thermo Fischer Scientific, Wilmington, DE, EEUU).

Genotipado. El análisis del genotipo de IGF2, MC4R, PRKAG3 y PEPCK-C se llevó a cabo por PCR a tiempo real tal y como se describe en Burgos et al (10), Burgos et al (11), Galve et al (12) y Latorre et al (1) respectivamente.

\section{RESULTADOS}

En la tabla 1 se muestra el resultado de los análisis del genotipo de IGF2 de los cerdos Pampa Rocha y de las razas autóctonas de España y Portugal. El alelo G es el alelo salvaje. El alelo A representa en la población de Piétrain el $99 \%$ de los alelos totales. Por el contrario, los cerdos Pampa Rocha son casi todos homocigotos GG. Tan solo uno de los animales es heterocigoto. En este sentido, los jabalís, las razas y líneas de cerdos españoles 
y los cerdos portugueses Bízaro son muy similares a los Pampa Rocha: los cerdos Porco Celta y los cerdos de las dos líneas ibéricas analizadas son todos homocigotos GG y tan solo dos cerdos Bízaro son heterocigotos, siendo el resto de ellos homocigotos GG.

Tabla 1. Genotipos y frecuencias alélicas de IGF2 de todos los animales analizados.

\begin{tabular}{lccccc}
\hline & \multicolumn{3}{c}{ Genotipos IGF2 } & \multicolumn{2}{c}{$\begin{array}{c}\text { Frecuencias } \\
\text { alélicas }\end{array}$} \\
\cline { 2 - 6 } & GG & AG & AA & G & A \\
\hline Jabalí & 6 & 0 & 0 & 1.000 & 0.000 \\
Ibérico & & & & & \\
$\quad$ Andalucía & 25 & 0 & 0 & 1.000 & 0.000 \\
$\quad$ Extremadura & 24 & 0 & 0 & 1.000 & 0.000 \\
Porco Celta & 35 & 0 & 0 & 1.000 & 0.000 \\
Bízaro & 50 & 2 & 0 & 0.981 & 0.019 \\
Pampa Rocha & 59 & 1 & 0 & 0.992 & 0.008 \\
Piétrain & 0 & 1 & 97 & 0.005 & 0.995 \\
\hline
\end{tabular}

El resultado del análisis del genotipo de MC4R aparece recogido en la tabla 2 . No resulta para nada sorprendente que el alelo A esté prácticamente fijado en los cerdos Piétrain, una raza caracterizada por una baja ingesta de alimento (13). Sí resulta curioso, por el contrario, que los jabalís también presenten este mismo genotipo. Esto mismo sucede con todas las razas de la península ibérica, a pesar de que en todos los casos la presencia de alelos A dista mucho de ser insignificante. Los genotipos MC4R de los cerdos Pampa Rocha difieren considerablemente de los de todas estas razas y líneas ya que en ellos los alelos más abundantes son los alelos $A$.

Tabla 2. Genotipos y frecuencias alélicas de MC4R de todos los animales analizados.

\begin{tabular}{lccccc}
\hline & \multicolumn{3}{c}{ Genotipos MC4R } & \multicolumn{2}{c}{$\begin{array}{c}\text { Frecuencias } \\
\text { alélicas }\end{array}$} \\
\cline { 2 - 6 } & GG & AG & AA & G & A \\
\hline Jabalí & 6 & 0 & 0 & 1.000 & 0.000 \\
Ibérico & & & & & \\
$\quad$ Andalucía & 23 & 2 & 0 & 0.960 & 0.040 \\
$\quad$ Extremadura & 20 & 4 & 0 & 0.917 & 0.083 \\
Porco Celta & 33 & 2 & 0 & 0.971 & 0.029 \\
Bízaro & 39 & 13 & 0 & 0.875 & 0.125 \\
Pampa Rocha & 8 & 32 & 21 & 0.393 & 0.607 \\
Piétrain & 91 & 7 & 0 & 0.964 & 0.036 \\
\hline
\end{tabular}

En la tabla 3 se muestran los genotipos PRKAG3. El alelo presumiblemente salvaje, el alelo G, es el predominante $(97 \%)$ en los jabalís. Las líneas ibéricas tienen mayoritariamente alelos A (90-100\%). Los cerdos Pampa Rocha y Porco Celta tienen ligeramente más alelos A (61 \% aproximadamente) que G (39\% aproximadamente). Los cerdos Piétrain y Bízaro tienen, sin embargo, ligeramente más alelos $\mathrm{G}$ que alelos $\mathrm{A}$.

Por último, en la tabla 4 se muestran los genotipos PEPCK-C. En esta tabla no están incluidos los cerdos Bízaro y Porco Celta porque, lamentablemente, cuando descubrimos este SNP (1) no teníamos disponible DNA de cerdos de estas razas. En todas las razas y líneas no seleccionadas por contenido magro, incluida la Pampa Rocha, predomina el alelo A. En el extremo opuesto se encuentran los cerdos Piétrain, con más alelos $C$ que alelos $A$.

Tabla 3. Genotipos y frecuencias alélicas de PRKAG3 de todos los animales analizados.

\begin{tabular}{lccccc}
\hline & \multicolumn{3}{c}{ Genotipos PRKAG3 } & \multicolumn{2}{c}{$\begin{array}{c}\text { Frecuencias } \\
\text { alélicas }\end{array}$} \\
\cline { 2 - 6 } & GG & AG & AA & G & A \\
\hline Jabalí & 15 & 1 & 0 & 0.969 & 0.031 \\
Ibérico & & & & & \\
$\quad$ Andalucía & 0 & 5 & 20 & 0.100 & 0.900 \\
$\quad$ Extremadura & 0 & 0 & 11 & 0.000 & 1.000 \\
Porco Celta & 7 & 13 & 15 & 0.386 & 0.614 \\
Bízaro & 9 & 21 & 2 & 0.609 & 0.391 \\
Pampa Rocha & 3 & 9 & 7 & 0.395 & 0.605 \\
Piétrain & 8 & 7 & 1 & 0.719 & 0.281 \\
\hline
\end{tabular}

Tabla 4. Genotipos y frecuencias alélicas de PEPCK-c de todos los animales analizados.

\begin{tabular}{lccccc}
\hline & \multicolumn{3}{c}{ Genotipos PEPCK-C } & \multicolumn{2}{c}{$\begin{array}{c}\text { Frecuencias } \\
\text { alélicas }\end{array}$} \\
\cline { 2 - 6 } & CC & AC & AA & C & A \\
\hline Jabalí & 0 & 3 & 10 & 0.115 & 0.885 \\
Ibérico & & & & & \\
$\quad$ Andalucía & 0 & 8 & 9 & 0.235 & 0.765 \\
$\quad$ Extremadura & 0 & 0 & 9 & 0.000 & 1.000 \\
Pampa Rocha & 1 & 8 & 30 & 0.128 & 0.872 \\
Piétrain & 21 & 26 & 2 & 0.694 & 0.306 \\
\hline
\end{tabular}

\section{DISCUSION}

El alelo A de IGF2, originado en cerdos asiáticos, es responsable del $10-20 \%$ de la variabilidad fenotípica en los caracteres grasos (5). Se trata, por lo tanto, de un SNP de gran relevancia dados sus enormes efectos fenotípicos sobre caracteres morfológicos y productivos importantes $(10,14)$. Es uno de los pocos marcadores que puede utilizarse por sí mismo como criterio de selección. Su uso en Pampa Rocha está limitado por la práctica ausencia de alelos A. Los alelos A están asociados tanto a valores reducidos en el espesor de tocino dorsal como a contenidos de IMF elevados. Si se pretendiese implementar un esquema productivo consistente en cruzar hembras Pampa Rocha (mayoritariamente GG) con machos Duroc $A A$, el status actual de IGF2 en los cerdos Pampa Rocha podría resultar muy adecuado. Esto es así dado que el locus de IGF2 se encuentra sujeto a imprinting (15); tan solo se expresan en la descendencia los genes recibidos por vía paterna, haciendo que el alelo materno resulte irrelevante para la $\mathrm{F} 1$. Sin embargo, este alelo $\mathrm{G}$ tiene una gran importancia para los caracteres maternales; de hecho, el genotipo GG en las hembras da lugar a 0.5-1 lechones más en cada parto (16).

Este hecho es consecuencia del enorme efecto de engrasamiento que produce el alelo $\mathrm{G}$ y de la conocida relación existente entre un adecuado engrasamiento de las hembras y una mayor capacidad de producción de leche, como consecuencia de lo cual se produce un aumento el número de lechones vivos en la descendencia. 
MC4R codifica un receptor implicado en los circuitos neuronales que regulan la ingesta de alimento $(17,18)$. El alelo A está asociado a un incremento de la ingesta de alimento $y$, como consecuencia de ello, a un incremento en BT y en la velocidad de crecimiento $(6,19,20)$. Utilizando este SNP como criterio de selección puede dirigirse ésta en cerdos Pampa Rocha para favorecer el engrasamiento (alelos A) o las carnes magras (alelos G). Como los cerdos Pampa Rocha ya presentan un nivel de engrasamiento suficientemente elevado, pensamos que podría ser recomendable seleccionar animales con el genotipo GG para mejorar los caracteres productivos de esta raza.

El mecanismo por el que el SNP en este gen descrito por Ciobanu et al (7) tiene efectos sobre los caracteres grasos no ha sido todavía elucidado. PRKAG3 codifica una subunidad reguladora de la proteína quinasa activada por $A M P$, que se encuentra implicada en la determinación del contenido en glucógeno del músculo estriado y, como consecuencia de ello, en el pH final de la carne. El alelo $A$ se encuentra asociado a valores elevados de $B T(12,21)$. La selección de alelos A o $G$ es una decisión complicada ya que afecta a distintos caracteres fenotípicos de una manera compleja. Sin embargo, la presencia prácticamente equilibrada de alelos G y A de este SNP hace que su selección en Pampa Rocha resulte un proceso sencillo.

Por último, el alelo $\mathrm{A}$ es el alelo asociado a un mayor contenido de grasa intramuscular, a un menor BT y a una exudación más baja (1), un fenotipo extraordinariamente valioso y poco común para la producción de carne de gran calidad. Esto significa que la carne de los cerdos Pampa Rocha podría mejorarse eliminando de los esquemas reproductivos los pocos animales que contienen alelos $C$.

El escaso número de muestras analizadas no nos permite realizar un estudio de haplotipos combinando los cuatro genes analizados. En nuestro estudio no hemos considerado un gen mayor extraordinariamente relevante, RYR1, por su implicación en la aparición de carnes PSE (22), aunque es bastante poco probable encontrar variabilidad en este marcador dado que esta mutación se originó presumiblemente en la raza Piétrain.

Los cerdos Pampa Rocha presentan frecuencias alélicas similares a las de las razas autóctonas de la península ibérica para algunos genes asociados a procesos de mejora. Esto es especialmente cierto en el caso de IGF2, cuyo alelo G está fijado en todas las razas o líneas no sometidas a selección artificial, incluida la Pampa Rocha, mientras que, por el contrario, el alelo A está prácticamente fijado en los cerdos Piétrain, el paradigma de muscularidad. Las frecuencias alélicas para el marcador PEPCK-C en todas las razas no seleccionadas presentan un patrón similar a pesar de que existe cierta variabilidad con respecto a este marcador en todas las razas analizadas. Pampa Rocha presenta unas frecuencias alélicas de PRKAG3 bastante semejantes a la de los cerdos de origen céltico, y en especial en el caso de los Porco Celta. Por el contrario, Pampa Rocha difiere de todas las razas autóctonas estudiadas en este trabajo en cuanto a las frecuencias alélicas del marcador MC4R, lo que demuestra la singularidad de esta raza derivada probablemente de su diferente historia evolutiva.

\section{Conflicto de intereses}

Los autores no declaramos ningún conflicto de intereses.

\section{Agradecimientos}

Este trabajo ha sido financiado por el proyecto AGL201566177-R concedido a Pascual López-Buesa. Silvia Lambí agradece la financiación recibida de CSIC-UdelaR.

\section{REFERENCIAS}

1. Latorre $\mathrm{P}$, Burgos $\mathrm{C}$, Hidalgo J, Varona $\mathrm{L}$, Carrodeguas JA, López-Buesa, P. C.A2456C-substitution in Pck1 changes the enzyme kinetic and functional properties modifying fat distribution in pigs. Sci Rep. 2016; 6:19617. https://doi.org/10.1038/ srep19617

2. Font-i-Furnols M, Tous N, Esteve-Garcia E, Gispert $M$. Do all the consumers accept the marbling in the same way? The relation between visual and sensory acceptability of pork. Meat Sci. 2012; 91(4):448-453. https://doi.org/10.1016/j. meatsci.2012.02.030

3. Reina R, López-Buesa $P$, Sánchez del Pulgar J, Ventanas J, García C. Effect of IGF-II (insulinlike growth factor-II) genotype on the quality of dry-cured hams and shoulders. Meat Sci. 2012; 92(4):562-568. https://doi.org/10.1016/j. meatsci.2012.05.027
4. Pugliese C, Sirtori F. Quality of meat and meat products produced from southern European pig breeds. Meat Sci. 2012; 90(3):511-518. https:// doi.org/10.1016/j.meatsci.2011.09.019

5. Van Laere AS, Nguyen M, Braunschweig M, Nezer C, Collete $C$, Moreau $L$ et al. A regulatory mutation in IGF2 causes a major QTL effect on muscle growth in the pig. Nature. 2003; 425(6960):832-836. https://doi.org/10.1038/nature02064

6. Kim KS, Larsen N, Short T, Plastow G, Rothschild MF. A missense variant of the melanocortin-4receptor (MC4R) gene is associated with fatness, growth, and feed intake traits. Mam Genome. 2000; 11(2):131-135. https://doi.org/10.1007/ $\underline{\mathrm{s} 003350010025}$ 
7. Ciobanu D, Bastiaansen J, Massoud M, Helm J, Woollard J, Plastow G, Rothschild M. Evidence for new alleles in the protein kinase adenosine monophosphate-activated g3-subunit gene associated with low glycogen content in pig skeletal muscle and improved meat quality. Genetics. 2001; 159(3):1151-1162. PMID: 11729159

8. Montenegro M, Llambí S, Castro G, Barlocco N, Vadell A, Landi V et al. Genetic characterization of Uruguayan Pampa Rocha pigs with microsatellite markers. Genet Mol Biol. 2015; 38(1):48-54. https://doi.org/10.1590/s1415-475738120140146

9. Fuentes V, Ventanas S, Ventanas J, Estévez M. The genetic background affects composition, oxidative stability and quality traits of Iberian dry-cured hams: Purebred Iberian versus reciprocal Iberian $\times$ Duroc crossbred pigs. Meat Sci. 2014; 96(2):737-743. https://doi.org/10.1016/j.meatsci.2013.10.010

10. Burgos C, Galve A, Moreno C, Altarriba J, Reina R, García C, López-Buesa. The effects of two alleles of IGF2 on fat content in pig carcasses and pork. Meat Sci. 2012; 90(2):309-313. https://doi. org/10.1016/j.meatsci.2011.07.016

11. Burgos C, Carrodeguas JA, Moreno C, Altarriba J, Tarrafeta L, Barcelona JA et al. Allelic incidence in several pig breeds of a missense variant of pig melanocortin-4 receptor (MC4R) gene associated with carcass and productive traits; its relation to IGF2 genotype. Meat Sci. 2006; 73(1):144-150. https://doi.org/10.1016/j.meatsci.2005.11.007

12. Galve A, Burgos C, Varona L, Carrodeguas JA, LópezBuesa P. Allelic frequencies of PRKAG3 in several pig breeds and its technological consequences on a Duroc 9 Landrace-Large White cross. J Anim Breed Genet. 2013; 130(5):382-393. https://doi. org/10.1111/jbg. 12042

13. Saintilan R, Mérour I, Schwob S, Sellier P, Bidanel J, Gilbert H. Genetic parameters and halothane genotype effect for residual feed intake in Piétrain growing pigs. Livest Sci. 2011; 142(1-3):203-209. https://doi.org/10.1016/j.livsci.2011.07.013
14. Van den Maagdenberg K, Stinckens A, Claeys E, Buys N, De Smet S. Effect of the insulin-like growth factor-II and RYR1 genotype in pigs on carcass and meat quality traits. Meat Sci. 2008; 80(2):293-303. https://doi.org/10.1016/j.meatsci.2007.12.008

15. Li C, Bin Y, Curchoe C, Yang L, Feng D, Jiang Q et al. Genetic imprinting of $\mathrm{H} 19$ and IGF2 in domestic pigs (Sus scrofa). Anim Biotechnol. 2008; 19(1):22-27. https://doi.org/10.1080/10495390701758563

16. Stinckens A, Mathur $P$, Janssens $S$, Bruggeman V, Onagbesan OM, Schroyen $M$ et al. Indirect effect of IGF2 intron3 g.3072G > A mutation on prolificacy in sows. Anim Genet. 2010; 41(5):493-498. https:// doi.org/10.1111/j.1365-2052.2010.02040.x

17. Krashes MJ, Lowell BB, Garfield AS. Melanocortin-4 receptor-regulated energy homeostasis. Nat Neurosci. 2016; 19(2):206-219. https://doi. org/10.1038/nn.4202

18. Shen WJ, Yao T, Kong X, Williams KW, Liu T. Melanocortin neurons: Multiple routes to regulation of metabolism. Biochim Biophys Acta. 2017; 1863(10 Pt A):2477-2485. https://doi. org/10.1016/j.bbadis.2017.05.007

19. Davoli R, Braglia S, Valastro V, Annaratone C, Comella M, Zambonelli et al. Analysis of MC4R polymorphism in Italian Large White and Italian Duroc pigs: association with carcass traits. Meat Sci. 2012; 90(4):887-892. https://doi.org/10.1016/j. meatsci.2011.11.025

20. Switonski M, Mankowska M, Salamon S. Family of melanocortin receptor (MCR) genes in mammalsmutations, polymorphisms and phenotypic effects. J Appl Genet. 2013; 54(4):461-472. https://doi. org/10.1007/s13353-013-0163-z

21. Škrlep $M$, Kavar $T$, Santé-Lhoutellier $\mathrm{V}$, ČandekPotokar M. Effect of I199V polymorphism at PRKAG3 gene on carcass and meat quality traits in Slovenian commercial pigs. J Muscle Foods. 2009; 20(3):367-376. https://doi.org/10.1111/j.17454573.2009.00158.x

22. Salas RC, Mingala C. Genetic Factors Affecting Pork Quality: Halothane and Rendement Napole Genes. Anim Biotechnol. 2017; 28(2):148-155. https:// doi.org/10.1080/10495398.2016.1243550 